\title{
CONTRIBUTIONS CONCERNING THE INFLUENCE OF THE RUNNING-IN ON THE ROUGHNESS OF THE SURFACES WITH PLANE CONTACT OF THE FRICTION COUPLE
}

\author{
Virgil Florescuㄴ, Florin Liviu Isvoranu², Constantin Tiganesteanu ${ }^{2}$, Liliana-Laura Badita ${ }^{3}$ \\ ${ }^{1}$ University of Civil Engineering, Bucharest, Romania \\ 2Institute of Solid Mechanics of the Romanian Academy, Bucharest, Romania \\ ${ }^{3}$ National Institute of Research and Development in Mechatronics and Measurement Technique, Bucharest, Romania \\ E-mails:florescuvirgil@yahoo.com, florinisvo@yahoo.com, tctin2002@yahoo.com, badita l@yahoo.com
}

\begin{abstract}
The running-in is usually regarded as a process that is continually modifying properties of the two contact surfaces during the initial phase of machine parts operation. This study is focused to analyse the dynamic behaviour of tribological wear system, based on equations system. The wear dependence, wear rate and roughness were chosen based on mathematical considerations. However, coefficients and exponents were introduced to close the model curves of wear encountered in real cases. Two state equations of the system were set: one is the wear equation on the effect of roughness on the wear rate; the other is changing the equation of mean square deviation $\sigma$ that occurs during the wear process. A study of the influence of the optimal roughness and stable roughness on running-in was made.
\end{abstract}

Keywords: Running-in, Wear, Plane Contact, Optimal Roughness, Equilibrium Roughness.

\section{Introduction}

Running-in is usually regarded as a process in which the properties of two contact surfaces are continuously and monotonously altered during the initial stage of the machinery parts (friction couple) operation. From the systemic theory point of view, running-in can be considered as a process of selfadaptation and adjustment of a tribological system consisting of two surfaces in contact with friction and the environment between them. It comes out that running-in is a process in which a dynamic system converts from an unbalanced state to a stable state.

So, the system has a precise limitation of its ability to adapt and adjust. Some authors characterize the running-in as mild wear or as "zero wear" $[4,15,16]$. For example, when the load applied to the system exceeds a critical value, the process of self-adjusting the system state will change from converging to divergence and, thus, will suffer severe wear. This dynamic wear, convergence or divergence, of tribological systems does not only occur at the initial stage of the machinery parts operation, but can also occur at any time when the balance of the system is destroyed for some reason.

The dynamic behaviour of the tribological system depends on its response. It may be geometric, physical, or chemical. The study of the surface topography effects on the dynamic behaviour of tribological systems at wear and running-in is presented.

\section{Response characteristics of tribological systems}

The paper subject is a lubricated plane/plane type friction couple (Figure 1).

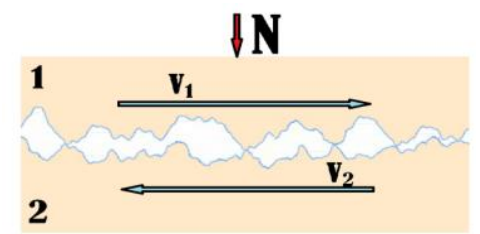

Figure 1: Lubricated plane/plane friction couple

The most important variable describing wear on this tribological system is the wear volume $W$. For fluid or mixed friction, the average film thickness $h$ can be chosen as another status variable. However, when comparing it with the wear volume $W$, the variation of $h$ during running-in is relatively small as the load, slip speed and lubricant are maintained the same. In an initial system, $h$ is treated as a constant that leads to a mono-variable first-order wear system. Wear rate $\dot{W}=d W / d t$ is dependent on the surface properties such as geometrical, physical and chemical characteristics, which are successively modified. Thus, they can be expressed as a function of the wear volume $W$. A self-adjusting loop in the tribological system is born (Figure 2) [12]. 


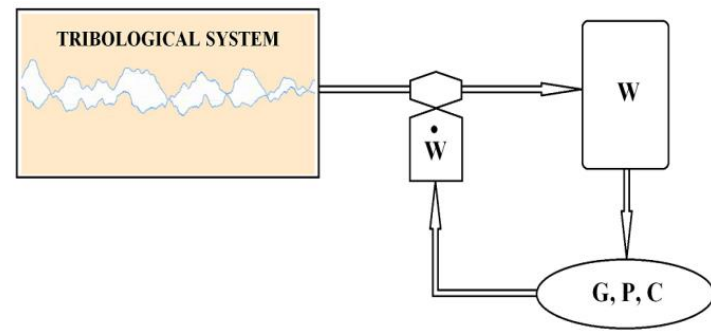

Figure 2: Self-adjusting loop of tribological system.

A system status equation can be written as:

$$
\dot{W}=F\{G(W) ; P(W) ; C(W)\}
$$

where $G(W) ; P(W) ; C(W)$ are expressions representing geometric, physical and chemical effects.

The above equation can be rewritten as:

$$
\dot{W}=f(W)
$$

$f(W)$ is the wear function.

It must be specified that the wear rate will never be able to have negative values. For a preliminary use of dynamic wear, it is convenient to develop the function $f(W)$ as a Taylor series at $W=0$ :

$$
\dot{W}=a_{0}+a_{1} \cdot W+a_{2} \cdot W^{2}+\cdots
$$

where: $a_{0}=f(0) ; a_{1}=f^{\prime}(0) ; a_{2}=f^{\prime \prime}(0) / 2 \ldots$

The system can be approximated by retaining two or three terms to the right of the equation (3). Such a first-order system was studied by Li in 1990 [10] and was not dealt with further. However, some characteristics observed in the wear and running-in processes shall be explained by such systems, based on the effect of surface roughness, on the dynamic behaviour of the systems subjected to wear. For the most complete model, several physical variables have been taken in consideration of the system.

\section{The state equation for wear systems, considering the effect of surface roughness}

It is well known that the surface topography changes continuously during aging processes, affecting the wear rate of the system. In order to understand the role of the derivation of a law of wear rate change and surface roughness, the surface roughness parameter should be considered as an independent status variable, which together with the wear volume $W$, constitutes a two-order wear system with two variables.

The system state equation should first answer two questions:

- How does the roughness of the surface affect the wear rate?

and

- How is the roughness changed in the wear processes?
The study objectives consist in a dynamic behaviour of the tribological system at wear analyse, based on the system equations, to establish a relationship between roughness and wear.

As it is known, for a full description of the surface topography, there are several parameters, including the amplitude parameter, the height density distribution function, the slope and the curvature radius of the roughness, all these being related to the wear behaviour. However, for a simple dynamic wear model, it is impossible to include all simultaneous effects of the various parameters. The problem is to choose the parameter that is most representative in terms of its effect on the aging speed and the most appropriate as a system status variable.

In many studies, the commonly used parameters [14] are either the ratio $\sigma^{*} / r,\left(\sigma^{*}\right.$ is the standard deviation of the peak distribution and $r$ represents the average radius of the peaks) $[1,5,11,20,21]$ or $\operatorname{tg} \theta$ representing the absolute average slope of the surface roughness [19]. This choice may be theoretically reasonable, but it is mathematically uncomfortable.

The authors of this paper consider that the rootmean-square deviation of the roughness $\left(R_{q}\right)$ is a representative parameter of the surface, in relation to the wear rate and as a system status variable. The reasons for this choice are:

- the need to establish a system status equation, initially covering only one area parameter;

- the changes in the roughness amplitude parameters during the running-in period are mostly fully investigated so far as are the changes in the radius of curvature and slope of the asperity. However, they are insufficient for a mathematical description (mainly because of uncertainty in measurement);

- for a given rough surface in a wear process, the modifications of the parameters mentioned are not independent of each other, the change in the curvature radius and the pick slope being reflected in the amplitude parameters variation.

For the study, the assumptions consist in:

- the two surfaces of the presented system have three-dimensional roughness;

- the upper surface is hard and unused during the wear process (or used very slowly compared to the counterweight);

square deviation of the roughness $\left(R_{q}\right)$ remains unchanged.

- the counterweight is considered to be the surface with the lowest hardness and which is subjected to wear, having a squared average roughness $\left(R_{f}\right)$.

Considering $R_{\max 1}$ and $R_{\max 2}$, the maximum profile heights of the two surfaces in contact, Kimura and Sugimura used the difference in the heights $\left(R_{\max 1}-\right.$ 
$\left.R_{\max 2}\right)$ and profile coordinates $\left(y_{1}-y_{2}\right)$ as a composite roughness.

The root-mean-square deviation of the composite profile $(\sigma)$ is:

$$
\sigma^{2}=R_{f}^{2}+R_{q}^{2}-2 R_{f} \cdot R_{q} \cdot C_{r}
$$

where $\left(C_{r}\right)$ is the coefficient of correlation between the contact surfaces.

In the event that the two surfaces are independent, we have $C_{r}=0$ and $\sigma^{2}=R_{f}{ }^{2}+R_{q}{ }^{2}$.

For fully compliant surfaces, we have $C_{r}=1, R_{f}=$ $R_{q}$ and $\sigma=0$.

The compound roughness does not only reflect the modifications of the two surfaces, but also contains the important information of correlation around it (compliance), with a substantial effect on the wear rate [6]. Therefore, the $\sigma$ is used here to characterize changes in the surface microgeometry and its influence on the wear.

\section{The roughness effect on wear rate - wear equation}

The wear rate, corresponding to the various wear mechanisms, can cover a few orders of magnitude. The way in which various factors, including roughness, affect wear will be different for the various wear-out mechanisms. The wear process under investigation is that corresponding to the lubricated slip friction with a non-linear wear rate, such as in the case of running-in. Some researchers describe this type of wear as "severe wear" (Quinn), this in terms of the process intensity, and others as "soft wear" (Whitehouse), in terms of its effects. It is considered that in this area of wear, the dominant mechanisms are abrasive and adhesive wear [13, 19], where roughness has a strong effect on wear rate $[1,2,9,17,18]$. In the case of abrasive wear and for some materials under conditions of adhesive wear, a very well-known wear equation has been formulated:

$$
W=K \cdot \frac{N \cdot L}{C \cdot H}
$$

in which: $W=$ wear volume; $N=$ normal loading; $L=$ slip distance; $H=$ hardness; $K=$ wear coefficient; $C=$ geometric constant, having the value 1 for abrasion and value 3 for adhesion friction.

The wear coefficient $K$ is usually considered to have a constant value when wear rate of the system is constant. For the dynamic wear, the greatest interest is the way in which the coefficient $K$ varies together with the wear rate.

By derivation with respect to time the equation (5), becomes:

$$
\dot{W}=\frac{N \cdot v}{H} \cdot F(W, \sigma)
$$

where $\dot{W}=\mathrm{d} W / \mathrm{d} t$, and $v=$ relative sliding speed between contact surfaces; $\sigma=$ the mean-square deviation of the composite roughness; $F(W, \sigma)=\mathrm{a}$ function representing the effect of roughness and physico-chemical factors on wear.

Changes in the physico-chemical properties of the system during the wear process are important factors affecting its rate. However, the mathematical modelling of these changes and their effects is a challenge. The effort to establish a real dynamic system model for running-in would have had a chance of success today if all aspects had been taken into consideration. In addition, in some cases and at each stage of the wear process, mechanical factors, such as the roughness or film thickness, may dominate the wear process and the physico mechanical effects may be out of it. There are many studies on the effects of roughness on the wear. At this initial stage, the physical and chemical aspects of the systems are assumed unchanged during the wear process, so only the root-mean-square deviation of roughness is considered as a variable factor. The $F(W, \sigma)$ function can be reduced to a $F(\sigma)$ function.

The wear rate will increase as the roughness amplitude increases for most materials in abrasion and adhesive processes. However, for very smooth surfaces, wear rate will increase with decrease of roughness $[3,15,16]$.

Using molecular-mechanical friction theory, Kragelsky obtains an equation for determining the friction coefficient as a function of the roughness parameter.

$$
f=A \cdot \Delta^{-\frac{v}{2 v+1}}+\beta+B \cdot \Delta^{\frac{v}{2 v+1}}
$$

where: $f=$ friction coefficient; $\Delta=$ roughness parameter; $v=$ parameter of the load curve; $\beta=$ the hardness factor of the molecular bonding; $A, B=$ constants related to the physico-chemical properties of materials, normal pressure and adhesive properties of the surface layers interaction.

It has also been noted by Kragelsky that for fatigue friction phenomenon, the connection between wear and friction may be expressed as: $I_{h} \approx$ $f t$, where $I_{h}$ is the wear rate and $t$ is a universal parameter for such types of defects and varies within the range $(2 \div 12)$ [9].

Therefore, the ratio $v / 2 v+1$ has values in the range $(1 / 3 \div 1 / 2)$, while varies between $(1 \div \infty)$. The exponent $n_{0}=\frac{v}{2 v+1}$ is considered as a constant, during the process of wear. The relationship between wear and friction can be written as follows:

$$
I_{h} \approx\left(D \cdot \Delta^{-n \sigma}+\beta+B \cdot \Delta^{n \sigma}\right)^{t}
$$

According to Kragelsky's theory and from equation (6), a simplified expression for the effect of the function $F(\sigma)$ can be written:

$$
F(\sigma)=a_{0}+\frac{a_{1}}{\sigma^{n_{1}}}+a_{2} \cdot \sigma^{n_{2}}
$$


where:

$n_{1}, n_{2}$ are indices that depend on the material and the type of wear; $n_{I}$ determining is a challenge. For the present study it was considered $n_{1}=1, n_{2}=2$.

$a_{0}, a_{1}, a_{2}$ are coefficients depending on the physicochemical factors (such as the material and the type of friction - limit, mixed, fluid).

Finally, the wear equation, for sliding motion wear, in the limit lubrication regime, becomes:

$$
\dot{W}=P \cdot\left(a_{0}+\frac{a_{1}}{\sigma}+a_{2} \cdot \sigma^{2}\right) ; P=\frac{N \cdot v}{H}
$$

$P$ is defined as an operating parameter, and the expression in parentheses corresponds to the wear coefficient $K$ in equation (5).

It is well known that there are two major mechanisms that lead to the variation of surface topography, although the roughness changes in a very complex way during the wear process:

1 : The cutting process of the asperities' tops. It is considered a smooth plane, which is sliding on a softer rough surface.

2: Adjustment of surfaces during the wear process. And in this case, there are two processes that result in the modification of the surface topography:

- the running-in, after which the surfaces become smooth and compliant;

- micro-grinding of the surface, after which the surfaces are affected due to the appearance of the wear particles, the transfer of material, the adhesions, the effects of fatigue, etc.

There are few theories that establish a relationship between these processes and surface damage. As a first approximation, to be able to take into account the effect of surface adjustment, suppose that the modification of the surface topography is proportional to the wear rate $\dot{W}$ and an operational coefficient $P$. Often it is necessary to make a distinction between the different wear processes:

- a gentle wear process, which can be independent of the surface roughness;

- a severe wear process, proportional to the surface roughness. The surface will be more damaged as the value of $\sigma$ increases.

The speed of its deterioration will be expressed by:

$$
(d \sigma / d t)_{q}=\left(b_{1} \cdot \sigma \cdot P+b_{2} \cdot P\right) \cdot W
$$

where the coefficients $b_{1}, b_{2}$ represents the probabilities that the surfaces will be worn and depend on the type of lubrication and other physicochemical factors.

According to equation (10) the wear rate will never be equal to zero, but it is expected that the surface will remain in a stable, unchanged state (that is, the parameters that characterize the topography of the surface to have constant values) under the conditions in which the wear rate reaches the lowest value.

Therefore, it can be substituted $\dot{W}$ in expressions (10) and (11) with $\left(\dot{W}-\dot{W}_{m}\right)$, where $\dot{W}_{m}$ is the minimum wear rate. Expressions (5) and (6) lead to obtain the variation equation of the mean-square deviation of the surface roughness in the wear process:

$$
d \sigma / d t=(d \sigma / d t)_{1}+(d \sigma / d t)_{2}
$$

meaning,

$$
d \sigma / d t=\left(-b_{0} \cdot \sigma+b_{1} \cdot \sigma \cdot P+b_{2} \cdot P\right) \cdot\left(\dot{W}-\dot{W}_{m}\right)
$$

\section{Simulation of the Dynamic Behaviour of the Slid Wear System}

Equations (10) and (13) constitute a nonlinear dynamic system.

Numerical integration of the wear state equation is basic. The values of $W$ and $\sigma$ at times $t$ and $t+\Delta t$ are given by $W_{j} ; \sigma$ and $W_{j+1} ; \sigma_{j}+1$. The differential equations (10) and (13) can be approximated by:

$$
\begin{gathered}
W_{j+1}=W_{i}+P \cdot\left(a_{0}+a_{1} / \sigma_{j}+a_{2} \cdot \sigma_{j}^{2}\right) \cdot \Delta t \\
\sigma_{j+1}=\sigma_{j}+\left(-b_{0} \cdot \sigma_{j}+b_{1} \cdot \sigma_{j} \cdot P+b_{2} \cdot P\right) \cdot\left(\dot{W}_{j}-\dot{W}_{m}\right) \cdot \Delta t
\end{gathered}
$$

where $\dot{W}_{j}=\left(W_{j}-W_{j-1}\right) / \Delta t, j=0,1,2$.

$W$ and $\sigma$ are determined for an arbitrary time using equations (14) and (15) for an initial value $W_{0}$ and $\sigma_{0}$. $W_{0}$ can be zero.

Coefficients $a_{0}, a_{1}, a_{2}$ and $b_{0}, b_{1}, b_{2}$, can be done experimentally. For example, can be specified:

1. $a_{0}$ is considered zero because it affects the wear rate through a constant and has no influence on the surface subjected to the wear process;

2. it is well known that this wear coefficient $K$ varies in the range of $10^{-2}-10^{-7}$. For values of $K$ $=10^{-4}$, and $\sigma=0,1, a_{2}$ (for $K=a_{2} \cdot \sigma^{2}$ ) becomes $0,01\left(1 / \mu \mathrm{m}^{2}\right)$;

3. if $a_{1} / \sigma$ and $a_{2} \cdot \sigma^{2}$ temperature fluctuates around the same value, we can have $a_{1} \approx a_{2} \cdot \sigma^{3}$. Considering $\sigma$ between 0,01 and $0,3 \mu \mathrm{m}$, we adopt $a_{1}=10^{-5} \cdot a_{2}$;

4. $b_{0}$ determines the time scale. The authors adopt arbitrary $b_{0}=0,1$. Significant values are for $b_{1} / b_{0}$ and $b_{2} / b_{0}$; their effects on the behaviour of the system will be presented in the next paragraph.

The theoretical determination will be the subject of future in-depth research. The curves, time - wear and time - roughness are useful in explaining the dynamic phenomena of running-in and wear. 
Discussions are limited to the case of limit lubrication, and the approach of the running-in under the mixed lubrication conditions for future studies remains.

\section{Optimal roughness and equilibrium roughness}

The running-in can often lead to a equilibrium roughness, independent of the initial roughness, a fact confirmed by many studies and researches $[4,7$, 8]. The wear system expressed by equations (10), (13), define a similar behaviour.

The balance of a dynamic system is reached when the state variables of the system have fixed and constant values over time. In practice it is impossible for the wear to disappear completely, or the wear volume to remain constant. Therefore, the system is defined as reaching its balance point when $\mathrm{d} \sigma / \mathrm{d} t=0$ and remains equal to zero all the time. According to equation (13), there are two balance points for the wear system:

$$
\left(\dot{W}-\dot{W}_{m}\right)=0 \text { and }\left(-b_{0} \cdot \sigma+b_{1} \cdot P \cdot \sigma+b_{2} \cdot P\right)=0
$$

Equality $\left(\dot{W}-\dot{W}_{m}\right)=0$ expresses that the wear rate can reach a minimum for a given surface roughness. This roughness is defined as the optimum roughness, $\sigma_{p}$. Its expression results from the derivation of equation (10), with:

$$
\sigma_{p}=\left(\frac{a_{1}}{2 a_{2}}\right)^{\frac{1}{3}}
$$

The roughness corresponding to the other equilibrium point is defined as the equilibrium roughness, $\sigma_{e}$, and results from the expression (16):

$$
\sigma_{e}=\frac{b_{2} \cdot P}{b_{0}-b_{1} \cdot P}=\frac{b_{2}}{b_{0}} \cdot \frac{P}{\left(1-\frac{P}{P_{e}}\right)}
$$

where $P_{e}=b_{0} / b_{1}$.

$\sigma_{p}$ and $\sigma_{e}$ represent two different concepts.

The optimum potential state is based on the $\sigma_{p}$, while $\sigma_{e}$ represents a possible condition of the system. In accordance with equation (10), coefficients $a_{1}$ and $a_{2}$ represent the roughness possibilities to reduce or increase the wear rate. The optimal roughness $\sigma_{p}$ corresponds to the balance of these two effects. According to equation (13), $b_{0}$ and $b_{2}$ describe the possibilities of wear processes to retouch the roughness or scratch the surface. So, the equilibrium roughness $\sigma_{e}$ represents the balance between smoothing and surface damage during running-in process. Obviously, $\sigma_{e}$ will increase with the increase in $b_{2} / b_{0}$ ratio. The curves in Figure 3 show the variation of the wear volume $W$ and the current roughness $\sigma$ over time $(t)$. It can be seen from Figure 3 that $\sigma$ is possible to tend asymptotically towards different values, which strongly depend on the value of the $b_{0} / b_{2}$ ratio.



(a)

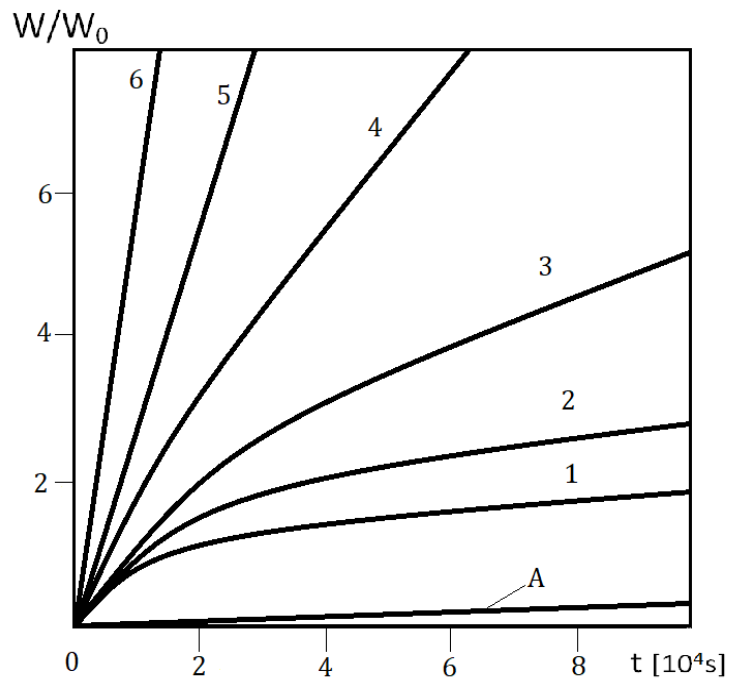

(b)

Figure 3: The variation of $\sigma(a)$ and $W(b)$ as a function of time and dependence on the ratio $b_{2} / b_{0}$

For the present system, according to the relation (17), the optimal roughness is $0,022 \mu \mathrm{m}$ and $a_{1} / a_{2}=$ $10^{-5}$. In the case of A (dotted line), where the initial roughness $\sigma_{0}=0,001 \mu \mathrm{m}$, that $\sigma_{0}<\sigma_{p}$, the roughness $\sigma$ converges to the optimal value $\sigma_{p}$.

For curves $1-5$, where $\sigma_{0}=0,3 \mu \mathrm{m}$, namely $\sigma_{0}>$ $\sigma_{\mathrm{p}}$, the system converges to the equilibrium roughness $\sigma_{e}$ if $b_{2} / b_{0}$ is not so small; $\sigma_{e}<\sigma_{p}$ when the value of the equilibrium roughness, calculated from equation (18), is much smaller than the optimal roughness, the system will converge to the optimal state $\sigma_{p}$ (curve 1 , for example).

In conclusion, from the ones presented, it turns out that there is an optimal roughness for a wear system, $\sigma_{p}$, which may be different from the equilibrium roughness produced by a running-in process.

Reaching the optimum state by means of the running-in can be achieved either by improving the 
running conditions by reducing the equilibrium roughness $\sigma_{e}$ or, if this is impossible, by reducing the original roughness $\sigma_{0}$. On the other hand, equation (18) shows that the equilibrium roughness $\sigma_{e}$ will be affected by the operating parameter $P$. If $P_{c}=b_{0} / b_{1}=$ 10 , the operating parameter $P$ changes from 1 to 2.6 $\left(\mathrm{cm}^{3} / \mathrm{s}\right)$. Figure 4 shows that equilibrium roughness $\sigma_{e}$ and curves $\sigma-t$ increase asymptotically with increasing $P$.

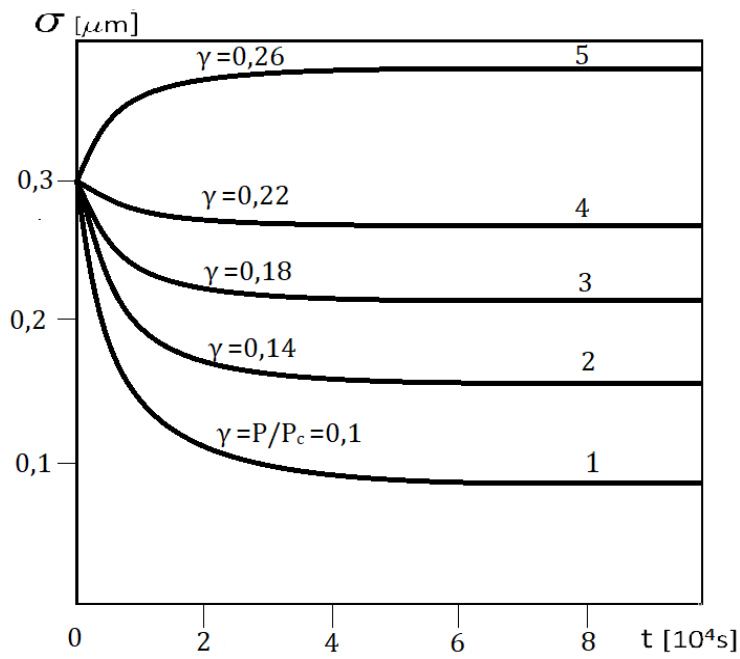

(a)



(b)

Figure 4: Dependence of the curves $\sigma$ - $t(a)$ and $W-t(b)$, on the parameter $P$

\section{Stability}

From equation (18) it is observed that the $\sigma_{e}$ value increases when the parameter $P$ increases continuously for a given $b_{2} / b_{0}$ ratio. When $P=P_{c}, \sigma_{e}$ will tend to infinity and the wear system will become unstable, which corresponds to a severe wear process. Figure 5 shows the simulated results for this process with $b_{2} / b_{0}=0,01 ; P_{c}=b_{0} / b_{1}=10$ and $\gamma=$ $P / P_{c}$ varying from 0,3 to 0,99 .

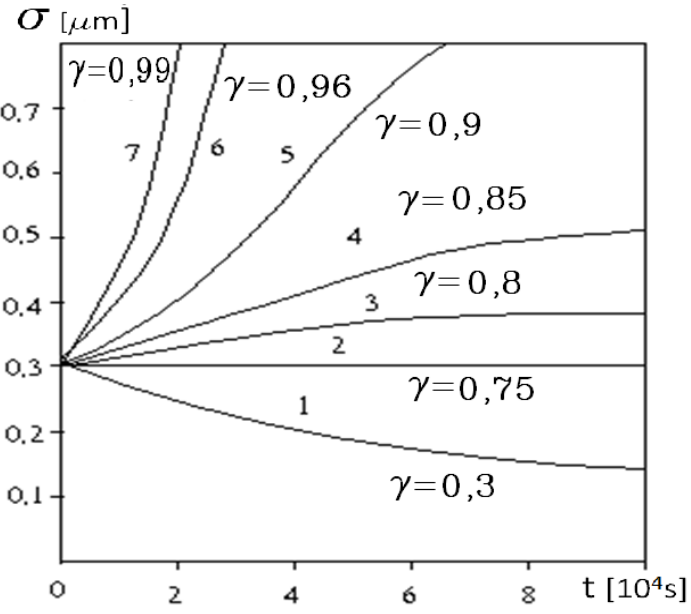

(a)

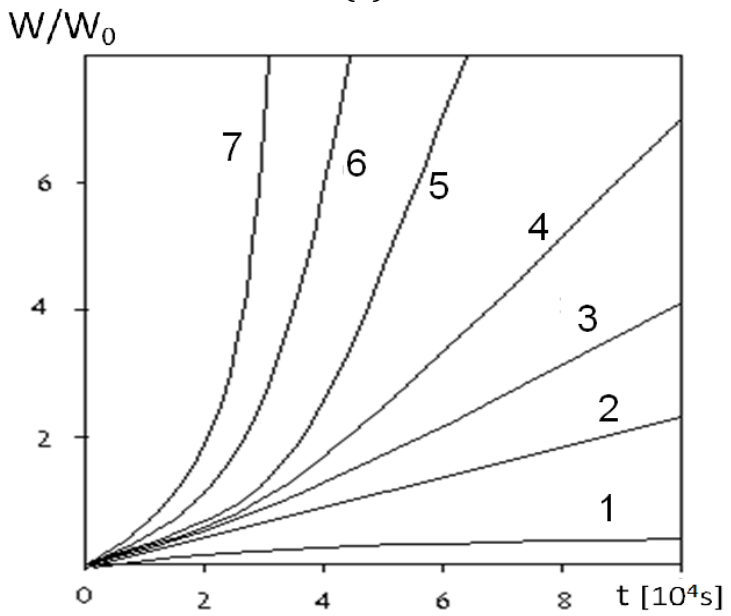

(b)

Figure 5: The influence of the operational parameter on the stability of the system

Stability properties depend only on the nature of the system around the equilibrium point. For the analysis it is mathematically convenient to replace the non-linear system by a linear system approximately close to the equilibrium point.

It is assumed that the system converges to the equilibrium roughness $\sigma_{e}$. A linear approximation for the nonlinear wear system will be:

$$
\begin{gathered}
\dot{W}=\dot{W}\left(\sigma_{e}\right)+\frac{d\left\{\dot{W}\left(\sigma_{e}\right)\right\}}{d \sigma \cdot\left(\sigma-\sigma_{e}\right)} \\
\dot{\sigma}=\dot{\sigma}\left(\sigma_{e}\right)+\frac{d\left\{\dot{\sigma}\left(\sigma_{e}\right)\right\}}{d \sigma \cdot\left(\sigma-\sigma_{e}\right)}
\end{gathered}
$$

These relationships can be written as follows:

$\dot{W}=\dot{W}\left(\sigma_{e}\right)+P \cdot\left(-\frac{a_{1}}{\sigma_{e}^{2}}+2 a_{2} \cdot \sigma_{e}\right) \cdot\left(\sigma-\sigma_{e}\right)$ 


$$
\dot{\sigma}=\left(-b_{0}+b_{1} \cdot P\right) \cdot\left[\dot{W}\left(\sigma_{e}\right)-\dot{W}_{m}\right] \cdot\left(\sigma-\sigma_{e}\right)
$$

It is easy to prove that this linear system has only one value of its own:

$$
\lambda=-b_{0} \cdot\left(1-\frac{P}{P_{c}}\right) \cdot\left[\dot{W}\left(\sigma_{e}\right)-\dot{W}_{m}\right]
$$

where: $P_{c}=b_{0} / b_{1}$.

According to the theory of linear systems stability, the necessary and sufficient condition for this equilibrium point to be asymptotically stable is $\lambda$ $<0$, that is $P<P_{c}$.

The system is marginally stable if $P=P_{c}$ and unstable if $P>P_{c}$. Therefore, $P_{c}$ is defined as the critical operating value at the equilibrium state of the wear system.

In a similar approximation, a nonlinear system approximation for the initial point is possible:

$$
\begin{aligned}
& \dot{W}=\dot{W}\left(\sigma_{0}\right)+P \cdot A_{0} \cdot\left(\sigma-\sigma_{0}\right) \\
& \dot{\sigma}=\dot{\sigma}\left(\sigma_{0}\right)+E \cdot\left(\sigma-\sigma_{0}\right)
\end{aligned}
$$

where

$$
A_{0}=\left(-\frac{a_{1}}{\sigma_{0}{ }^{2}}+2 a_{2} \cdot \sigma_{0}\right)
$$

and whose own value is:

$$
\begin{aligned}
& \lambda=E=P \cdot B_{0} \cdot\left(-b_{0}+b_{1} \cdot P\right)+ \\
& +P \cdot A_{0} \cdot\left(-b_{0} \cdot a_{0}+b_{1} \cdot P \cdot \sigma_{0}+b_{2} \cdot P\right)
\end{aligned}
$$

where:

$$
B_{0}=a_{1} \cdot\left(\frac{1}{\sigma_{0}} \cdot \frac{1}{\sigma_{p}}\right)+a_{2} \cdot\left(\sigma_{0}^{2} \cdot \sigma_{p}^{2}\right)
$$

It can be demonstrated that at the initial moment, before the start of the running-in, the system has a critical operational parameter:

$$
P_{C O}=P_{c} \cdot \frac{B_{0}+A_{0} \cdot \sigma_{0}}{B_{0}+A_{0} \cdot \sigma_{0}+A_{0} \cdot\left(\frac{b_{2}}{b_{1}}\right)}
$$

It results from the ones shown above, that, for the examined system, having a sliding speed, the running will increase the critical loading capacity with the factor:

$$
\frac{P_{c}}{P_{C O}}=1+\frac{b_{2}}{b_{1}} \cdot \frac{A_{0}}{B_{0}+A_{0} \cdot \sigma_{0}}
$$

Equations (26), (28) and (30) show that the ratio $P_{c} / P_{c o}$ is independent of the parameter $P$, being influenced only by the initial roughness $\sigma_{0}$, the optimal roughness $\sigma_{p}$ and the coefficient $\left(b_{2} / b_{1}\right)$.

Figure 6 shows $\sigma-t$ and $W$ - $t$ curves of the wear system in Figure 5, but after running-in for a time $t_{0}$ $=80000 \mathrm{~s}$.
The dashed area shows the limit of the variation of the $\sigma-t$ and $W$ - $t$ curves when changing from 0,85 to 1,05 .

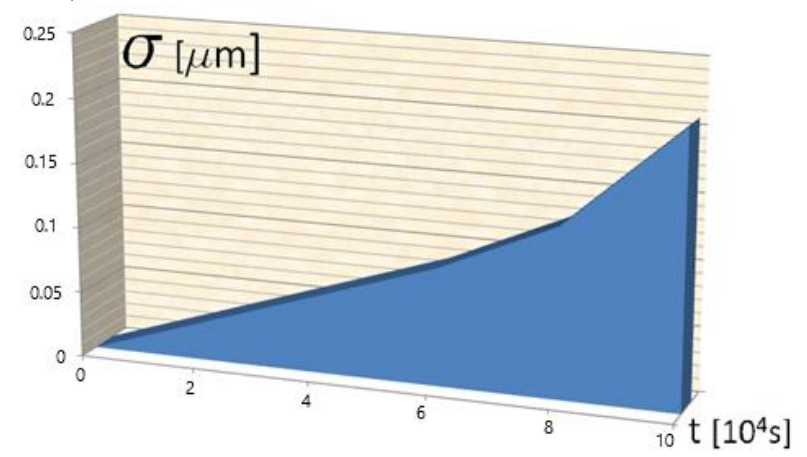

(a)

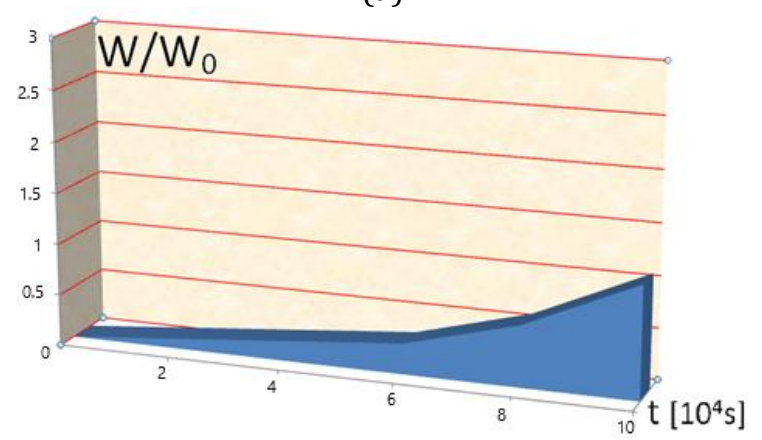

(b)

Figure 6: Curves $\sigma$-t (a), and $W$-t (b), after running-in.

Compared to figure 5, figure 6 shows clearly that if the same load is applied to the same system, after running-in the system will be much more stable than at first. It should be noted that in practice the increase in the load capacity during running-in will be much higher than the one calculated with equation (30) due to random material breaks which usually exist in the initial stage of the machinery components operation, and which has not been included in this model.

The operating parameter $P$ has been considered constant during wear. However, the running-in convergence may be achieved by applying a variable load, the optimum load curves may be predicted using the dynamic system model. This will be the subject of further studies.

\section{Conclusions}

Some assumptions that require further discussions have been made in this work. The form of dependence between wear, wear rate and roughness, was chosen on the basis of some mathematical considerations. However, coefficients and exponents have been introduced which bring this model closer to the wear curves encountered in real cases. 
Under these conditions the following requirements have been met:

1. The wear progresses constantly, but the wear rate is likely to change;

2. The amplitude of the initial roughness may be increased or decreased depending on the operating parameters and the running-in conditions;

3. A running-in process may be stable or unstable, depending again on running-in conditions.

All of these are characteristic of real processes, but it was clearly not possible to claim to obtain true quantitative results. However, from the wide spread of the curves, it was seen that it is possible to reduce the approximate values by carefully combining the parameters.

In order to apply this theoretical model on a much larger scale, a careful study of the physical relationships between different sizes is required. Such studies should probably include at least one experiment, so there is no generally valid theory for the wear mechanism. With these reservations, the model is considered useful as a guide in choosing optimal running-in programs.

In conclusion:

1. The running-in should be regarded as a selfadaptation process of a system, the dynamic behaviour of which is controlled by geometric, physical and chemical reactions. The effect of roughness on the dynamic behaviour of a lubricated slip-motion tribological system was analysed.

2. The root-mean-square deviation $\sigma$ of the composite roughness of two contact surfaces is considered as a parameter characterizing the effect of roughness on the wear. The parameter characterizes both surfaces and their conformity.

3. Two system state equations have been established: one is the wear equation for the effect of roughness on the aging speed; the other is the variation equation regarding the modification that takes place in the quadratic mean $\sigma$, during the wear process;

4. The equilibrium roughness $\sigma_{e}$ produced by a running-in process may not be equal to the optimum roughness $\sigma_{p}$, corresponding to a minimum system wear speed. A satisfactory running-in shall lead to the optimal condition, which be more achieved by reducing $\sigma_{e}$ or initial roughness ratio $\sigma_{0}$;

5. A critical operating value $P_{c}$ is derived from a wear system stability analysis. The system is stable for $P<P_{c}$, corresponding to running-in and unstable $P>P_{c}$, leading to severe wear.

\section{References}

[1] Albers, A., Reichert, R. (2017). On the influence of surface roughness on the wear behavior in the running-in phase in mixed-lubricated contacts with the finite element method. Wear, 376-377, Part B, 1185- 1193.

[2] Argatov, I.L., Fadin, Y.A. (2011). A macro-scale approximation for running-in period. Tribology Letter, 42, 311-217.

[3] Bayer, R.G., Sirico, J.L. (1975). The influence of surface roughness on wear. Wear, 35(2), 251260.

[4] Cabanettes, F., Rosén, B-G. (2014). Topography changes observation during running-in of rolling contacts. Wear, 315(1-2), 78-86.

[5] Georgescu, D., Rece, L., Ionescu, A., Apostu, A. (2019) Application of some evaluation methods associated with the performance of materials to determine the areas of use of concrete. Romanian Journal of Materials, 49(1), 148 - 156.

[6] Golden, J.M. (1976). The evolution of asperity height distribution of a surface subjected to wear. Wear, 39(1), 25-44.

[7] Jamari, J., Schipper, D.J. (2008). Deterministic repeated contact of rough surfaces. Wear, 264(34), 349-358.

[8] Jamari, J., Schipper, D.J. (2007). Deformation due to contact between a rough surface and a smooth ball. Wear, 262(1-2), 138-145.

[9] Kragelsky, J.V. (1982). Friction and wear, Pergamon Press, Oxford, pp 237-268, 297 - 316.

[10] Li, N. (1990) The characteristics of surface topography during running-in, $\mathrm{PhD}$ thesis, Tsinghua University, Beijing.

[11] Lorentz, B., Albers, A. (2013). A numerical model for mixed lubrication taking into account surface topography, tangential adhesion effects and plastic deformations. Tribology International, 59, 259-266.

[12] Masouros, G., Dimarogonas, A., Lefas, K. (1977). A model for wear and surface roughness transients during the running-in of bearings. Wear, 45(3), 375-382.

[13] Quinn, T.F.J. (1990). The classifications, laws, mechanisms and theories of wear, Fundamentals of Tribology, ed. by, Suh and Saka, MIT, pp 477492.

[14] Rece, L., Florescu, V., Modrea, A., Jeflea, V., Harničárová, M., Valíček, J., Borzan, M. (2020). Optimization of the $2 \frac{1}{2} \mathrm{D}$ processing method of complex parts, through a predictive algorithm for controlling the geometric shape deviations resulting from processing. Mathematics - MDPI - Open Access Journal, 8, 59. 
[15] Reizer, R., Pawlus, P., Galda, L., Grabon, W., Dzierwa, A. (2012). Modeling of worn surface topography formed in a low wear process. Wear, 278-279, 94-100.

[16] Reizer, R, Galda, L., Dzierwa, A., Pawlus, P. (2011). Simulation of textured surface topography during a low wear process. Tribology International, 44(11), 1309-1319.

[17] Reichert, S., Lorentz, B., Heldmaier, S., Albers, A. (2016). Wear simulation in non-lubricated and mixed lubricated contacts taking into account the microscale roughness. Tribology International, $100,272-279$.

[18] Reichert, S., Lorentz, B., Albers, A. (2015). Influence of flattening of rough surface profiles on the friction behaviour of mixed lubricated contacts. Tribology International, 93, 614 - 619.

[19] Whitehouse, D.J. (1990). The effects of surface topography on wear, Fundamentals of Tribology, ed. by, Suh and Saka, MIT, pp 17-52.

[20] Xue Zuo, Yu Tan, Yuankai Zhou, Hua Zhu, Haifeng Fang. (2018). Multifractal analysis of three-dimensional surface topographies of GCr15 steel and $\mathrm{H70}$ brass during wear process. Measurement, 125, 196-218.

[21] Yue, T., Wahab, M.A. (2017). Finite element analysis of fretting wear under variable coefficient of friction and different contact regimes. Tribology International, 107, 274-282. 